\title{
A Technology Acceptance Model to Design Evaluation for Health Management Terminal for Elderly in the Community
}

\author{
Sheng Zhou ${ }^{\mathrm{a}, 1}$, Jiecong Zong ${ }^{\mathrm{a}}$ and Qun $\mathrm{Wu}^{\mathrm{a}}$ \\ ${ }^{a}$ Zhejiang Sci-Tech University, Hangzhou, China, 310018
}

\begin{abstract}
Smart terminal for older adults is one of the core touchpoints of the service system, which connects older adults and public services provided by the community. Health Management Terminal for Older Adults in the Community (HMTOAC) is closely related to the health of older adults, and it is an important design issue. However, previous Health Management Terminals (HMT) are not designed for older adults. It is of great scientific significance and extensive application value to deeply explore the real demands of older adults. This research expanded the design process of "exploration-prediction-prototype-evaluation", and it took the needs of older adults as the starting point. Based on the Technology Acceptance Model (TAM), combined Structural Equation Model (SEM) and Technique for Order Preference by Similarity to Ideal Solution (TOPSIS), we investigated important influencing factors of HMTOAC design, and established the design evaluation model of the HMTOAC.
\end{abstract}

Keywords. Smart Terminal, Technology Acceptance Model (TAM), Technique for Order Preference by Similarity to Ideal Solution (TOPSIS), Older Adults

\section{Introduction}

There are 264 million people aged 60 and over, accounting for $18.70 \%$ of Chinese total population. The number of people aged 65 and above is 191 million, accounting for $13.50 \%$. Compared with the last National Census (2010), the proportions has increased by $5.44 \%$ and $4.63 \%$ respectively ${ }^{[1]}$. The aging of population is getting larger, thus increasing the stress for the family and society. To solve the problem, the formation and development of the community pension mechanism has gradually become a new trend of healthy pensions. Besides, there has been an increasing number of relevant policies. For example, The People's Government of Zhejiang Province proposed to stimulate new potentials for healthy consumption, and there were many deployments related to health management terminals ${ }^{[2]}$. At the same time, A survey in 2020 showed that the proportion of elder people using smart terminals to obtain medical and health information $(40 \%)$ had increased significantly, and it was an increase of $12 \%$ compared with 2019 . However, the aging of the elderly would lead to psychological and physical changes in the older adults ${ }^{[4-5]}$, and this would make the demand for information products of the elderly different from those of the young. These suggested that the elderly still had some

${ }^{1}$ Corresponding author: Qun Wu; Postal address: Zhejiang Sci-Tech University, Hangzhou, China, 310018; Email address: wuq@zstu.edu.cn. 
obstacles in the adoption and use of technology nowadays, such as the issues from cost, knowledge gap and privacy ${ }^{[3]}$. In the design and development process of the existing health management terminals, there is a lack of in-depth research on the different physiological and psychological needs of the elderly, and it makes the product lack of versatility. The age of aging, the needs of the elderly cannot be underestimated, so the research of the elderly community health management terminals (HMTOAC) appears to be quite essential. Unfortunately, the evaluation cycle of existing product design schemes is too long to obtain feedback from the elderly in time. After that, it is easy for the design positioning to lag behind the needs of old users, which affects the formulation of optimal design schemes.

The existing Decision-making (DM) methods could be roughly divided into three categories ${ }^{[6]}$ : (1) Multicriteria Decision Making (MCDM) technology, (2) Mathematical Programming (MP) technology and (3) Artificial Intelligence (AI) technology. Among them, TOPSIS is a method of Multicriteria Decision Making (MCDM) technology. The following are the advantages of TOPSIS: objective evaluation, clear calculation process and strong operability, and especially it can be flexibly combined with other analysis methods to establish a more accurate evaluation model. Compared with the previous single method or other combined methods, the combined evaluation method of SEM and TOPSIS can effectively improve the efficiency of plan formation and evaluation. In order to check the validity of the combined method, we chose a measurement index. Net Promoter Score (NPS) was a user satisfaction measurement index originally derived from the user loyalty research of Frederick Reichheld ${ }^{[10]}$, and it was based on a research hypothesis: only when users were truly satisfied with a certain product or service would they be willing to recommend it to friends or family. The higher the user experience scored, the higher the corresponding user increased multiple. It could be seen that NPS was a suitable tool for evaluating whether a product was satisfactory to users and whether the combined evaluation method of SEM and TOPSIS was useful.

In summary, from the perspective of design index research, most of the existing research is qualitative research, but there was almost no quantitative analysis of the subjective factors that hinder the use of technology by the elderly. On the other hand, from the perspective of design evaluation methods, there already are comprehensive evaluation methods have been proposed. But in the research on the design of the HMTOAC, those methods are not targeted at the elderly. There is still a lack of a rapid evaluation method for designers to have which is more in line with the conceptual design process. There is no doubt that the older adults have their own subjective perception of the community health management terminals, so the design research is carried out from it. After that, in order to explore the important factors influencing the design of the HMTOAC, the research uses a combination of Structural Equation Model (SEM) and Technique for Order Preference by Similarity to Ideal Solution (TOPSIS). The research provides theoretical support for the subsequent design and evaluation of the HMTOAC programs.

\section{Preliminaries}

\subsection{The definition of terminals service design}

In order to be more in line with the design and research of the HMTOAC, the interactive design methods in the MINDS service design methods had been changed to a smart 
product design method, and they were suitable for the combination of software and hardware. The Improved MINDS service design method is depicted as in Figure 1. The research analyzed the needs of various stakeholders in the design and research of community health management terminals from the three levels, included service concept, service system, and service experience.

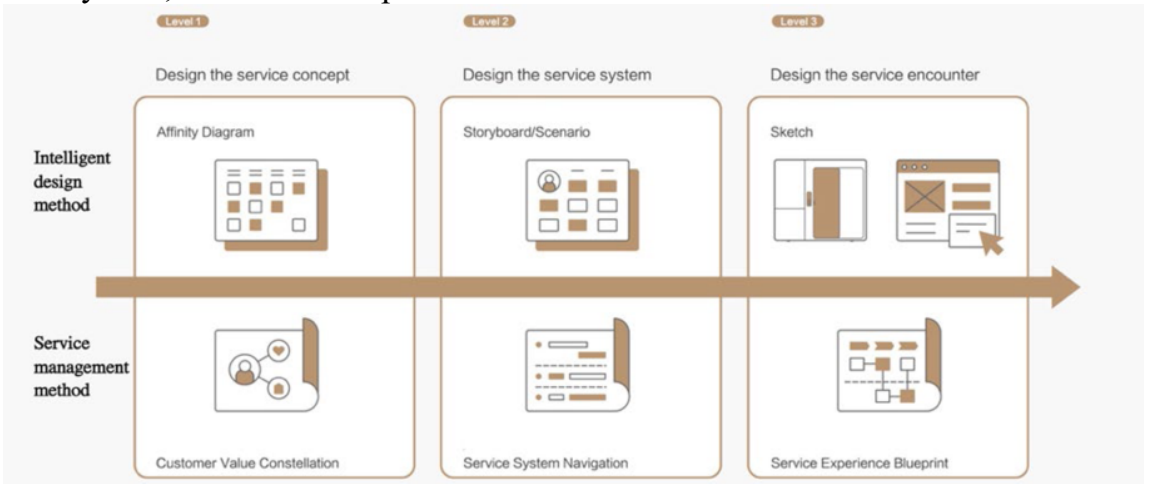

Figure 1. Model of Influencing Factors in the Design of the HMTOAC.

\subsection{The initial terminals design plan}

Most of the existing health management products were designed in two forms: a multifunction detection all-in-one machine or a small smart product plus a mobile APP design. But in the research, the appearance of the HMTOAC was designed as a cabin form, because of the public service attributes of future community scenarios. This appearance also provided a private inspection space for the older adults. In the independent cabin, the older adults could easily complete the health measurement under the guidance of the operation animation, even without deliberately learning medical knowledge. The elderly could get a health report at last. As long as those that meet the design strategy were ideal solutions, so there was more than one design solution. Design referenced GBT12985$1991^{[7]}$, GBT10000-1988 ${ }^{[8]}$ and GBT13547-1992 ${ }^{[9]}$. Figure 2 gives the initial design plan of four groups of HMTOAC.

Scheme I
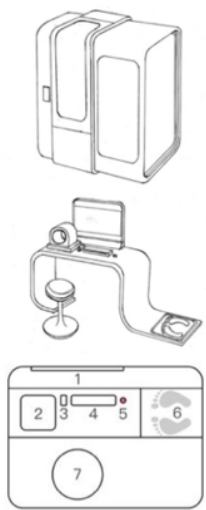

Scheme II
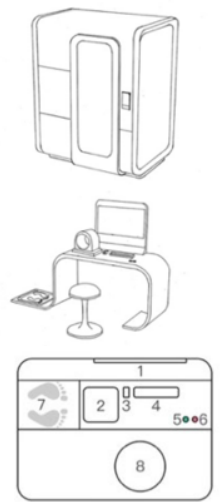

Scheme III
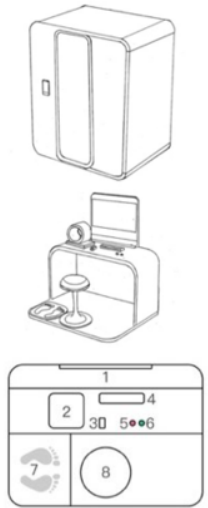

SchemeIV
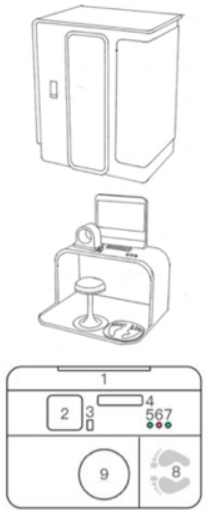

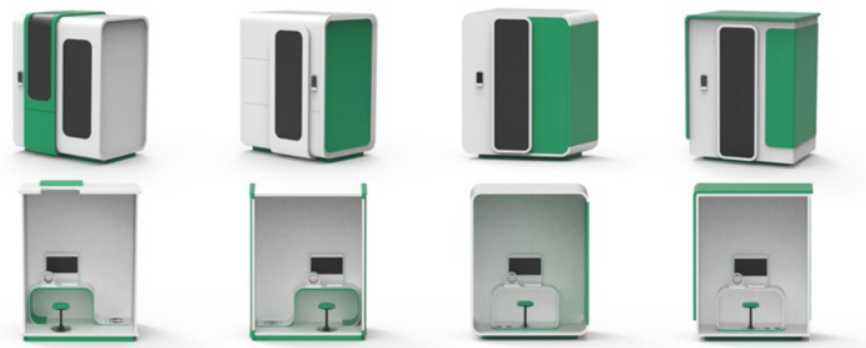

Figure 2. Initial design plan of the HMTOAC.

\subsection{Factors influencing the terminals design}

Based on the literature research on design criteria and technology acceptance theory, as well as preliminary investigations, the research hypotheses on the factors affecting the design of the HMTOAC were proposed. These hypotheses were based on six factors, and the factors included functional convenience, interaction suitability, structural reliability, spatial security, and modeling care, as well as sense and color matching comfort. The relationship between the six factors was the main hypotheses.

The collection of experimental data was through the questionnaires and interviews. After excluding untrue and incomplete questionnaires, there were a total of 437 valid questionnaires, with an effective rate of $90.29 \%$. Research used a variety of data analysis methods to analyze the differences in the influence of the above factors on the intention of the older adults to use the community health management terminals. After that, the following factors were significantly positively correlated with the influence of behavioral preferences of the elderly: functional convenience, interaction suitability, structural reliability, spatial security, and modeling care. And according to the results of high-level factor analysis, the top three influencing factors were functional convenience, spatial security, and modeling care.

Table 1. Design evaluation index weights of the HMTOAC

\begin{tabular}{cccccc}
\hline Index & $\begin{array}{c}\text { Functional } \\
\text { convenience }\end{array}$ & $\begin{array}{c}\text { Interaction } \\
\text { suitability }\end{array}$ & $\begin{array}{c}\text { Structural } \\
\text { reliability }\end{array}$ & $\begin{array}{c}\text { Sense of space } \\
\text { security }\end{array}$ & $\begin{array}{c}\text { Styling } \\
\text { care }\end{array}$ \\
\hline Weights & 0.292 & 0.088 & 0.078 & 0.334 & 0.208 \\
\hline
\end{tabular}

Based on the above content, the weights of indicators at all levels in the design and evaluation system of the community health management terminal were calculated. As shown in Table 1, the weight coefficients of the following factors were high: the index spatial security, functional convenience, and modeling care.

\section{Methods}

\subsection{TOPSIS evaluation model algorithm}

According to the above research content, the design evaluation model of the HMTOAC included 5 design indicators and 32 sub-items. At the same time, the four groups of community health management terminal initial design schemes were comprehensively evaluated by TOPSIS method. TOPSIS method is as follows: 
- Constructed the initial judgment matrix:

The method had $\mathrm{m}$ targets (objects) and $\mathrm{n}$ indicators (attributes), and the evaluation value of the $\mathrm{j}$-th index of the $\mathrm{i}$-th target is $x_{i j}$. So, the initial judgment matrix X was

$$
\mathrm{X}=\left[\begin{array}{cccc}
x_{11} & x_{12} & \cdots & x_{1 m} \\
x_{21} & x_{22} & \cdots & x_{2 m} \\
\vdots & \vdots & \ddots & \vdots \\
x_{n 1} & x_{n 2} & \cdots & x_{n m}
\end{array}\right]
$$

- Trending of evaluation indicators:

Before the calculation, the reciprocal method or the difference method was used to trend all indicators, even if the dependent variable changed in the same direction as the independent variable.

- Normalization of evaluation indicators

In order to ensure that the evaluation index values were calculated and compared within the same change range, the data matrix with the same change trend was normalized, and the corresponding matrix with the value range between 0 and 1 was established. The conversion formula was

$$
\mathrm{a}_{i j}=\frac{x_{i j}}{\sqrt{\sum_{i=1}^{n}\left(x_{i j}\right)^{2}}}
$$

In formula (2), $x_{i j}$ was the value of the $\mathrm{i}$-th evaluation target on the $\mathrm{j}$-th index after index convergence.

From this, the normalized matrix $\mathrm{A}$ is

$$
\mathrm{A}=\left[\begin{array}{cccc}
\mathrm{a}_{11} & \mathrm{a}_{12} & \cdots & \mathrm{a}_{1 m} \\
\mathrm{a}_{21} & \mathrm{a}_{22} & \cdots & \mathrm{a}_{2 m} \\
\vdots & \vdots & \ddots & \vdots \\
\mathrm{a}_{n 1} & \mathrm{a}_{n 2} & \cdots & \mathrm{a}_{n m}
\end{array}\right]
$$

- According to the A matrix, the positive ideal solution vector and the negative ideal solution vector were obtained:

The optimal solution $\mathrm{A}^{+}$was composed of the optimal value in each column of $\mathrm{A}$ :

$$
A^{+}=\left(\mathrm{a}_{i 1}^{+}, \mathrm{a}_{i 2}^{+} \ldots \mathrm{a}_{i n}^{+}\right)
$$

The worst scheme $\mathrm{A}^{-}$consisted of the worst value in each column of $\mathrm{A}$ :

$$
A^{-}=\left(\mathrm{a}_{i 1}^{-}, \mathrm{a}_{i 2}^{-} \ldots \mathrm{a}_{i n}^{-}\right)
$$

- Calculate the distance $\mathrm{D}_{i}^{+}$and $\mathrm{D}_{i}^{-}$between each evaluation target and the positive ideal solution and the negative ideal solution respectively:

$$
\begin{aligned}
& \mathrm{D}_{i}^{+}=\sqrt{\sum_{j=1}^{n}=\omega_{j}\left(\mathrm{a}_{i j}^{+}-\mathrm{a}_{i j}\right)^{2}} \\
& \mathrm{D}_{i}^{-}=\sqrt{\sum_{j=1}^{n}=\omega_{j}\left(\mathrm{a}_{i j}^{-}-\mathrm{a}_{i j}\right)^{2}}
\end{aligned}
$$

$\mathrm{D}_{i}^{+}$and $\mathrm{D}_{i}^{-}$were the distances between the $\mathrm{i}$-th evaluation target and the positive and negative ideal solutions, and $\mathrm{a}_{i j}$ was the value of the evaluation target $\mathrm{i}$ in the $\mathrm{j}$-th index. And $\omega_{j}$ was the weight coefficient of the indicator.

- Calculate the closeness $\mathrm{C}_{i}$ of each evaluation objective to the optimal solution, and sort the evaluation objectives:

$$
C_{i}=\frac{\mathrm{D}_{i}^{-}}{\mathrm{D}_{i}^{+}+\mathrm{D}_{i}^{-}}
$$


The evaluation objectives were ranked according to the size of $C_{i}$. The range of $C_{i}$ was between 0 and 1 . The larger the $C_{i}$, the better. In a series of evaluation objectives, the largest $C_{i}$ was the optimal solution, and the smallest $C_{i}$ was the worst solution.

\subsection{Evaluation of initial design}

Figure 3 shows a cardboard model for older participants to evaluate the community health management terminal design scheme after the test experience. A 5-point Likert scale was used to obtain user satisfaction ratings for the design scheme.

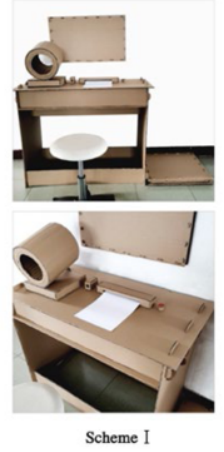

Scheme 1

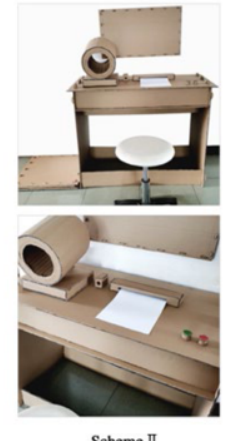

Scheme II

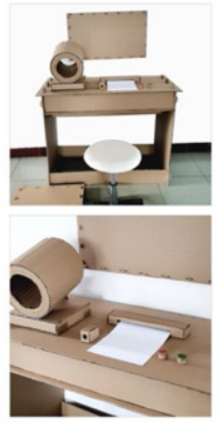

Scheme III

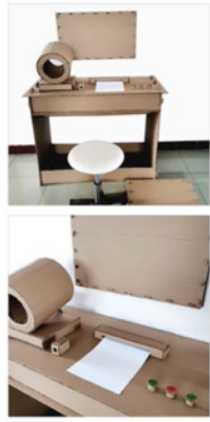

SchemeIV

Figure 3. Initial design plan cardboard model.

Randomly selected 17 senior citizens who met the requirements. Before actual operation, experimenters explained to participants all the functions and usage methods of the four groups of community health management terminal design schemes (numbered I, II, III, and IV). After participants were familiar with the operation of the equipment, they could then complete the inspection operation autonomously. The above operation were all in order to make sure that during the experiment operation, there was no error caused by the unfamiliarity of subjects, especially on the function and operation of the equipment. During the operation, the scores of the design indicators of each group of schemes were recorded.

After counting the valid questionnaire data collected by the subjects after using the cardboard model, we calculated and got the average scores of the four groups in each index. The results were shown in Table 2.

Table 2. Mean value of the evaluation index score for the design of the HMTOAC

\begin{tabular}{rrrrrr}
\hline Index & $\begin{array}{c}\text { Functional } \\
\text { convenience }\end{array}$ & $\begin{array}{c}\text { Interaction } \\
\text { suitability }\end{array}$ & $\begin{array}{c}\text { Structural } \\
\text { reliability }\end{array}$ & $\begin{array}{c}\text { Sense of space } \\
\text { security }\end{array}$ & Styling care \\
I & 4.147 & 3.627 & 4.044 & 3.908 & 3.899 \\
II & 4.221 & 4.078 & 4.176 & 4.000 & 3.950 \\
III & 3.993 & 3.951 & 4.250 & 4.311 & 4.303 \\
IV & 3.765 & 4.275 & 4.412 & 4.185 & 4.151 \\
\hline
\end{tabular}

There was determined the weight of the evaluation indicators for the design of the HMTOAC in preliminaries. After that, we ensured that the indicator change trend was the same, and after normalization of the sum of squares, a standardized matrix A was obtained, as shown in Table 3.

Table 3. Standardized evaluation matrix A of design evaluation index for HMTOAC

\begin{tabular}{rrrrrr}
\hline Index & $\begin{array}{c}\text { Functional } \\
\text { convenience }\end{array}$ & $\begin{array}{c}\text { Interaction } \\
\text { suitability }\end{array}$ & $\begin{array}{c}\text { Structural } \\
\text { reliability }\end{array}$ & $\begin{array}{c}\text { Sense of space } \\
\text { security }\end{array}$ & $\begin{array}{c}\text { Styling } \\
\text { care }\end{array}$ \\
\hline I & 0.514 & 0.455 & 0.479 & 0.476 & 0.478
\end{tabular}




\begin{tabular}{rrrrrr} 
II & 0.523 & 0.511 & 0.494 & 0.487 & 0.484 \\
III & 0.495 & 0.495 & 0.503 & 0.525 & 0.527 \\
IIII & 0.467 & 0.536 & 0.522 & 0.510 & 0.509 \\
\hline
\end{tabular}

We calculated the distance $\mathrm{D}_{i}^{+}$and $\mathrm{D}_{i}^{-}$between all the index values of each evaluation object and the positive and negative ideal solution, and the relative closeness $\mathrm{C}_{i}$ of each evaluation object to the positive ideal solution. The results were shown in Table 4. After testing and evaluation, among the four groups of community health management terminal design schemes for the elderly, scheme III was the best scheme.

Table 4. TOPSIS evaluation calculation results

\begin{tabular}{rrrrr}
\hline Design & $\begin{array}{c}\text { Positive ideal } \\
\text { solution distance }\end{array}$ & $\begin{array}{c}\text { Negative ideal } \\
\text { solution distance }\end{array}$ & $\begin{array}{c}\text { Relative } \\
\text { proximity C }\end{array}$ & Sort results \\
\hline I & 0.045 & 0.026 & 0.360 & 4 \\
II & 0.031 & 0.036 & 0.533 & 2 \\
III & 0.020 & 0.042 & 0.674 & 1 \\
IIII & 0.033 & 0.036 & 0.524 & 3 \\
\hline
\end{tabular}

\subsection{Optimize the initial design plan}

According to the TOPSIS evaluation and analysis results, the goal was to improve the factors, and the factors included convenience of functions, the suitability of interaction, the reliability of the structure, the sense of space security, and the sense of care for the shape. The optimization scheme III was to form a group of optimized design schemes for community health management terminals numbered $\mathrm{V}$.

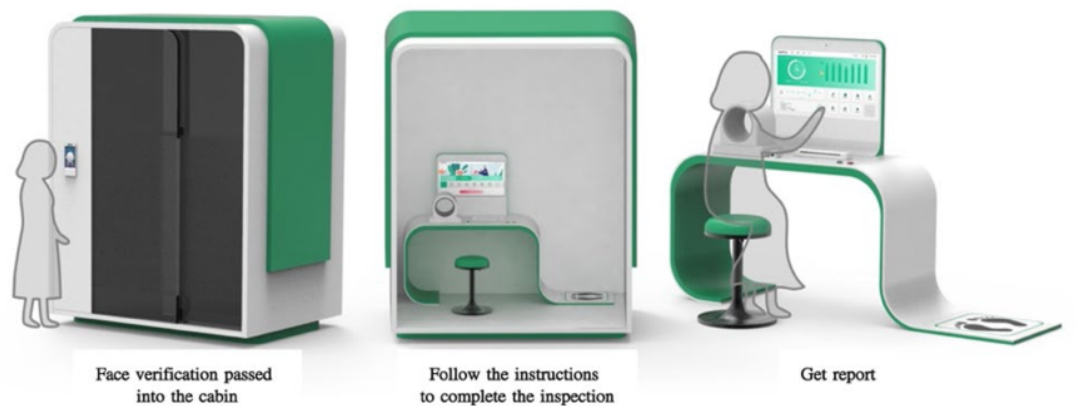

Figure 4. Optimized design plan V.

\subsection{NPS assessment method}

We recruited 21 expert users who met the positioning to score the optimized design plan and the aforementioned best design plan, and it is in order to verify the importance of design indicators for improving the satisfaction of the HMTOAC design. The overall satisfaction of users with the program was measured by a 10-point scale, and the scores of satisfactions with various design indicators of the program were evaluated by a 5point Likert scale. Besides, the net recommendation value was measured using an 11point scale. And the likelihood that users would recommend this product was done by asking a question: How likely are you to recommend the design to your family, friends or colleagues?

Figure 5 showed the calculation steps of NPS net recommendation value ${ }^{[11]}$ :

- Using a scale of 0 points (very unlikely) to 10 points (very possible) to ask customers whether they would recommend our products; 
- According to the scores, the respondents were divided into three categories:

a) Detractors: respondents with a score of 0 to 6 ;

b) Passives: respondents with a score of 7 or 8 ;

c) Promoters: respondents with a score of 9 or 10 ;

- Calculating the difference between the proportion of recommenders and the proportion of detractors, which was the NPS value.

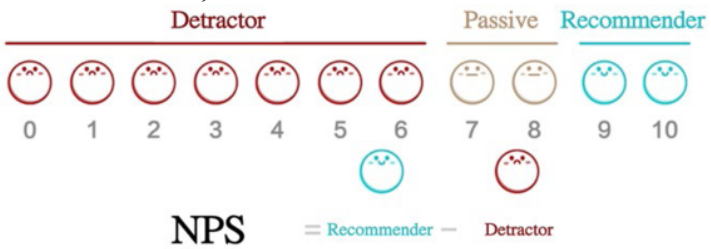

Figure 5. Net Promoter Calculation Method.

\section{Results}

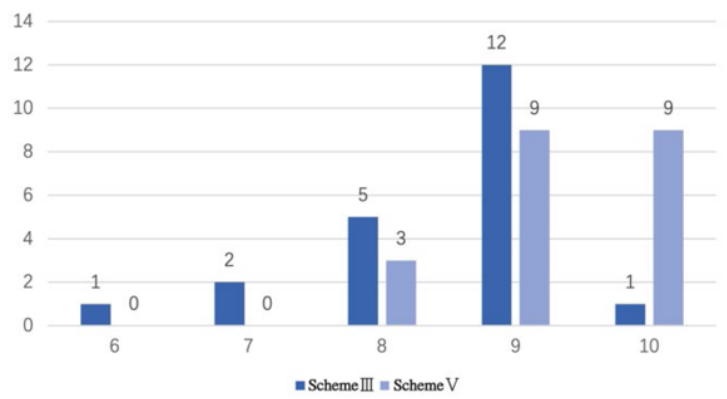

Figure 6. Scheme III and Scheme V User Recommendation Value Distribution.

To demonstrate the relative merit of the proposed method, we compared the user recommendation value and NPS between initial plan III and the optimized plan V. And we finally verified the validity of the design evaluation model. Figure 6 sketches these relationships. Among the 21 participants, there were totally 13 recommenders and 1 detractor in Scheme III, and there were totally 18 recommenders and 0 detractor in Scheme V. After calculating the NPS of Scheme III and Scheme V respectively, the results are as follows:

- $\quad$ NPS of Scheme III $=61.90 \%-4.76 \%=57.14 \%$

- $\quad$ NPS of Scheme V=85.71\%-0=85.71\%

It can be seen that the NPS of scheme V is much higher than that of scheme III, and it means the user loyalty and satisfaction of scheme $\mathrm{V}$ have been significantly improved after optimizing.

Table 5. Comparison of Satisfaction between Scheme III and Scheme V

\begin{tabular}{ccccr}
\hline Index & \multicolumn{2}{c}{ Satisfaction } & \multicolumn{2}{c}{ Importance } \\
\hline $\begin{array}{c}\text { Functional } \\
\text { convenience } \\
\begin{array}{c}\text { Interaction } \\
\text { suitability }\end{array}\end{array}$ & 4.131 & Scheme V & Scheme III & Scheme V \\
\hline
\end{tabular}




\begin{tabular}{rrrrr}
$\begin{array}{c}\text { Structural } \\
\text { reliability }\end{array}$ & 4.024 & 4.583 & 0.242 & 0.215 \\
$\begin{array}{r}\text { Sense of space } \\
\text { security }\end{array}$ & 4.143 & 4.558 & 0.200 & 0.481 \\
Styling care & 4.102 & 4.585 & 0.403 & 0.189 \\
\hline
\end{tabular}

The scheme III and scheme V satisfaction and importance numerical statistics are depicted as in Table 5. We took the mean of the satisfaction of each indicator as the independent variable, and took the correlation coefficient (it is also called importance) between the satisfaction of each indicator and the overall satisfaction of the scheme as the dependent variable. Then we analyzed the key driving factors of the design plan, and we obtain the key driving factors diagram as shown in Figure 6 finally.
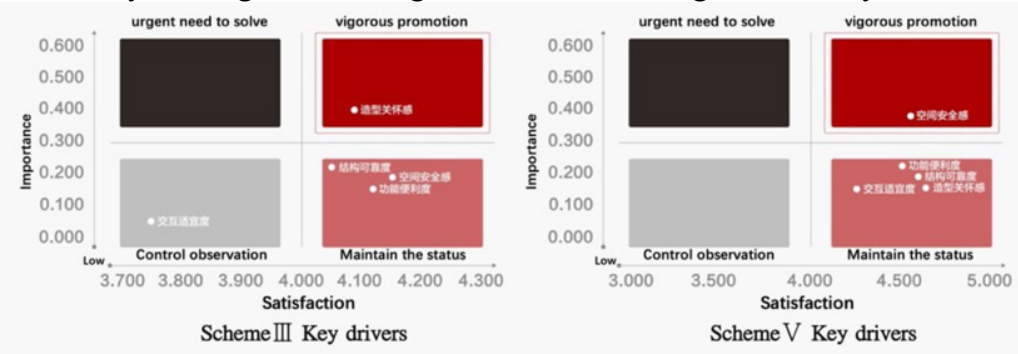

Figure 6. Analysis of Key Driving Factors of Scheme III and Scheme V.

After comparing the distribution of key driving factors in Scheme III and Scheme $\mathrm{V}$ in Figure 6, it can be seen that users are generally satisfied with the two schemes, so there are no factors in the "urgent need to solve" quadrant. Four of the five design indicators of Scheme III are at a higher level of satisfaction, indicating that users have a certain degree of recognition for Scheme III. At the same time, the styling care index of Scheme III is of higher importance, indicating that users are most satisfied with the styling care index of Scheme III. However, the satisfaction and importance of interaction suitability of Scheme III are low, so further optimization is needed. The satisfaction of the five design indicators of Scheme V is all higher than that of Scheme III. Besides, the spatial security of the design index of Scheme $\mathrm{V}$ is located in the quadrant of "vigorous promotion", which is the most recognized index by users.

\section{Conclusion}

The community health management terminals are closely related to the health status of the older adults, and the community health management terminal design is the current design research hotspot. In the digital information era, the integration of technology and design has become closer, which brings more possibilities for how to realize the needs of the elderly for design, and also brings a certain degree of cognitive barrier for the elderly to accept new technologies.

In this paper, we present a comprehensive design evaluation method which is able to effectively evaluate the community health management terminals. The research process followed the creative design process of "exploration-conception-prototypeevaluation", and it started with the mental and physical needs of the elderly for community health management terminal design. And the research analyzed the important factors influencing the design of the HMTOAC by using the method of combining SEM and TOPSIS. In this way, the design evaluation model of the HMTOAC was formed. 
Then from the four groups of initial design plans, we quickly judged the best plan. After analyzing the characteristics of the four groups of plans, an optimized design plan was formulated. Finally, the effectiveness of the optimized design plan and the design evaluation model was verified by comparing the user satisfaction of the optimized design plan and the aforementioned best design plan.

Although the research adopts the method of combining SEM and TOPSIS, and it applies this theory to the design of the HMTOAC. It is the novelty. But there are still some limitations due to various factors such as research geographical restrictions and insufficient research time, which includes:

(1) The sample acquisition is concentrated in the elderly community in Hangzhou, China. Although it is typical, it is necessary to consider the influence of regional factors such as different places of residence and different hometowns on the research results in the subsequent research;

(2) The sample data are cross-sectional data, not long-term follow-up surveys. Therefore, it is impossible to know the changes in the awareness of the elderly's needs for the design of community health management terminals over time.

In the future, with the upgrading of technology and the high attention to product design for the elderly at home and abroad, the research deficiencies will surely be overcome. The design of the health management terminal for the elderly community, and even the product design of the elderly in other scenarios in the future community will bring more well-being and create a better life for the elderly.

\section{Acknowledgment}

This work is supported by Zhejiang Province Philosophy and Social Science Planning Project (Grant No: 20NDJC084YB).

\section{References}

[1] National Bureau of Statistics of China, Seventh National Census in China, 2021.

[2] General Office of the People' s Government of Zhejiang Province, Implementation Opinions on Boosting Consumption and Promoting Economic Growth, 2020

[3] KAKULLA B. 2021 Tech Trends and the 50-Plus[R/OL]. AARP Research, 2021. https://doi.org/10.26419/res.00420.001.

[4] CHEN K, CHAN A H S. Geotechnology acceptance by elderly Hong Kong Chinese: a senior technology acceptance model (STAM)[J]. Ergonomics, 2014, 57(5): 635-652. DOI:10.1080/00140139.2014.895855.

[5] LI Q. Understanding Older Adults' Post-Adoption Usage Behavior and Perceptions of Mobile Technology[J]. International Journal of Design, 2018, 12(3): 93-110.

[6] CHAI J, LIU J N K, NGAI E W T. Application of Decision-Making Techniques in Supplier Selection: A Systematic Review of Literature[J]. Expert Systems with Applications, 2013, 40(10): 3872-3885. DOI: 10.1016/j.eswa.2012.12.040.

[7] The State Bureau of Quality and Technical Supervision, Apply the general rule of human body size percentile in product design, GBT12985-1991

[8] The State Bureau of Quality and Technical Supervision, Chinese adult body size, (1988-12-10), GBT10000-1988

[9] The State Bureau of Quality and Technical Supervision, Work space human body size standard, GBT13547-1992

[10] REICHHELD F F. The One Number You Need to Grow[J]. Harvard Business Review, 2003, 81(12): 46-54, 124.

[11] TULLIS T, ALBERT B, User experience measurement: collection, analysis and presentation, Electronic Industry Press, 2016 